\title{
A!
}

This is an electronic reprint of the original article.

This reprint may differ from the original in pagination and typographic detail.

Qiu, H.; Schneider, G. F.; Kauppinen, T.; Rudolph, S.; Steiger, S.

\section{Reasoning on human experiences of indoor environments using semantic web technologies}

\section{Published in:}

Proceedings of the 35th International Symposium on Automation and Robotics in Construction (ISARC 2018),

Berlin, Germany, July 20-25, 2018

DOI:

10.22260/ISARC2018/0013

Published: 01/01/2018

Document Version

Publisher's PDF, also known as Version of record

Please cite the original version:

Qiu, H., Schneider, G. F., Kauppinen, T., Rudolph, S., \& Steiger, S. (2018). Reasoning on human experiences of indoor environments using semantic web technologies. In Proceedings of the 35th International Symposium on Automation and Robotics in Construction (ISARC 2018), Berlin, Germany, July 20-25, 2018 (pp. 95-102). International Association on Automation and Robotics in Construction (IAARC).

https://doi.org/10.22260/ISARC2018/0013

This material is protected by copyright and other intellectual property rights, and duplication or sale of all or part of any of the repository collections is not permitted, except that material may be duplicated by you for your research use or educational purposes in electronic or print form. You must obtain permission for any other use. Electronic or print copies may not be offered, whether for sale or otherwise to anyone who is not an authorised user. 


\title{
Reasoning on Human Experiences of Indoor Environments using Semantic Web Technologies
}

\author{
H. Qiu ${ }^{a, d}$, G.F. Schneider ${ }^{a, d}$, T. Kauppinen ${ }^{b}$, S. Rudolph ${ }^{c}$ and S. Steiger ${ }^{d}$ \\ ${ }^{a}$ Technische Hochschule Nuremberg, Nuremberg, Germany \\ ${ }^{b}$ Aalto University School of Science, Finland \\ ${ }^{c}$ Technische Universität Dresden, Dresden, Germany \\ ${ }^{d}$ Fraunhofer Institute for Building Physics IBP, Nuremberg, Germany \\ E-mail: <firstname>.<lastname>@ibp.fraunhofer.de, sebastian.rudolph@tu-dresden.de, tomi.kauppinen@aalto.fi
}

\begin{abstract}
-
The Indoor Environmental Quality (IEQ) in a building affects occupants' well-being and productivity. Traditionally, models are developed to predict IEQ satisfaction from physical measurements. These approaches work fine in a laboratory environment but tend to fail in real-world applications. Recent work focuses on collecting direct human feedback on IEQ. However, existing approaches either lack the ability to capture the multiple dimensions of IEQ or the integration with other domain knowledge, e.g. from building information modeling or building automation systems. To tackle this problem, we have developed a novel approach based on Semantic Web Technologies (SWT) which enable interoperability and reasoning. In this paper, the HBC (Human Comfort in Building) ontology is presented, which formally specifies the domain of IEQ in multiple dimensions and relates it to adjacent domains. An online survey is designed in order to specify ontology requirements and collect human feedbacks for evaluating the ontology. We evaluate the use of the HBC ontology in two use cases in an office building: recommendation of spaces based on IEQ factors and recommendation of settings for technical equipment from collected feedbacks.
\end{abstract}

Keywords -

Human Experiences; Indoor Environmental Quality; Semantic Web Technologies; Ontology; Office Buildings

\section{Introduction}

In modern life, people spend between $80 \%$ and $90 \%$ of their time indoors [1]. Recent studies have shown that Indoor Environmental Quality (IEQ) not only can affect occupants' well-being, but also their productivity [2]. Dimensions, or factors, of IEQ are amongst others indoor air quality (IAQ), thermal comfort, visual comfort and acoustic comfort. In the past, researchers have identified a number of physical measurements which correlate with IEQ. In experiments, human satisfaction is determined from questionnaires while measuring physical quantities identified to correlate with IEQ (e.g. [3]). From the results, models have been developed to predict IEQ satisfaction from measurements.

However, since standards have often been found to be at odds with the requirements of occupants [4], recent work focuses on using direct human observations on IEQ with the aim to improve IEQ satisfaction of users [5, 6].

In the current state of art, several studies and tools exist to capture occupant experiences in buildings: Comfy [7], TherMOOstat [8], CrowdComfort [9], CBE Occupant Feedback Toolkit [10] and MYBUILDINGMESSAGE [11].

Comfy, TherMOOstat and CrowdComfort share a common downside: the inability to capture other IEQ factors besides thermal comfort. For CBE Occupant Feedback Toolkit and MYBUILDINGMESSAGE, although they can capture various IEQ dimensions, they lack the ability to integrate the occupants' experiences automatically with information from adjacent domains, e.g. Building Information Modeling (BIM).

There are also projects which try to capture occupants' experiences while integrating with adjacent domains. For instance, Twitter surveys have been used to integrate occupants' thermal comfort experiences [12]. YouSense is a web-based application which can capture human observations of spaces. It is built on the expressiveness of the EXPERIENCE vocabulary-a lightweight vocabulary for describing human experiences [6]. The authors argue that it is usable for linked data applications via collecting human experiences. However, it is notable that EXPERIENCE has a more general scope beyond IEQ factors.

To address these limitations of existing approaches, we developed an ontology that formally specifies human experiences on the several dimensions of IEQ in spaces. The ontology is aimed to derive a better understanding of perceived comfort, spaces, and relations between them. Further on, this ontology-based approach allows to infer new information given rules and linkages to information modeled according to BIM and BAS. We also designed a survey for collecting experimental data. The process of ontology development follows a well-known approach called METHONTOLOGY [13]. Semantic Web Technologies are used for ontology implementation, such as 
OWL DL and SWRL rules. The ontology is populated based experimental data using OWL API. We evaluated the ontology by means of SPARQL queries with regard to the targeted scenarios.

The rest of this paper is organized as follows. Section 2 describes the designed online human experience survey. Section 3 presents the HBC ontology and the development process of HBC. Section 4 shows the application of evaluation of HBC ontology in two use-cases. Section 5 discusses the limitation and future work. Section 6 provides the concluding remarks.

\section{Online Human Experience Survey}

We designed a human experience survey using an online questionnaire tool in order to gather data as an input for ontology development and validation.

There are thirteen questions that are about location (question 1), IEQ (question 2-5) and settings of building objects (question 6-13). Questions related to IEQ are the core parts. Apart from reviewing literature, we interviewed domain experts on common terms used. We designed questions and possible answers for assessing the IEQ aspects of human experiences.

Occupants can assess the quality of a certain IEQ as positive, neutral and negative. For instance, according to a person's experience, the thermal comfort can be assessed as positive. However, it is unknown whether the positiveness of thermal comfort is caused by feeling warm or cold. People can feel warm positively, or on the other hand cold positively due to differences in their age, culture or psychological factors. Therefore, we not only capture thermal comfort by quality, but also thermal sensation. In this way, thermal comfort can be assessed by the combination of them. Similar design choices were made for questions about acoustic comfort and visual comfort.

However, there is no duality for IAQ. Since, normally, people have a positive feeling when the air is fresh; when the air is stuffy, the quality assessment is negative.

The last part of the survey is about the settings of building objects. After finishing the questions about IEQ assessments, occupants have to report settings of building objects at that moment.

\section{Human Comfort in Buildings (HBC) On- tology}

\subsection{Ontology Engineering Methodology}

METHONTOLOGY [13] is an ontology engineering method we adopted for building the HBC ontology. It is widely used as it provides sufficient descriptions and allows ontology reuse [14]. It is an iterative process along with knowledge acquisition and evaluation as supporting activities. In this section we provide an overview of the steps of METHONTOLOGY.

\subsection{Specification}

The Specification stage consists of domain analysis and knowledge acquisition in the course of expert interviews and literature reviews. While designing the survey questions, the requirements of the ontology are identified which include the scenarios in which the ontology is used. Also a set of terms along with their characteristics are identified.

The HBC ontology models the scenario that an occupant describes about his/her experience at certain times in a space. Conceptually, the HBC ontology is divided into three modules: Hex ontology, bim4Hex ontology and Time ontology (see Figure 1). The module of Time describes necessary time concepts for the Hex ontology. Modularizing the HBC ontology is good for (1) reusing ontologies, (2) maintaining and extending ontologies.

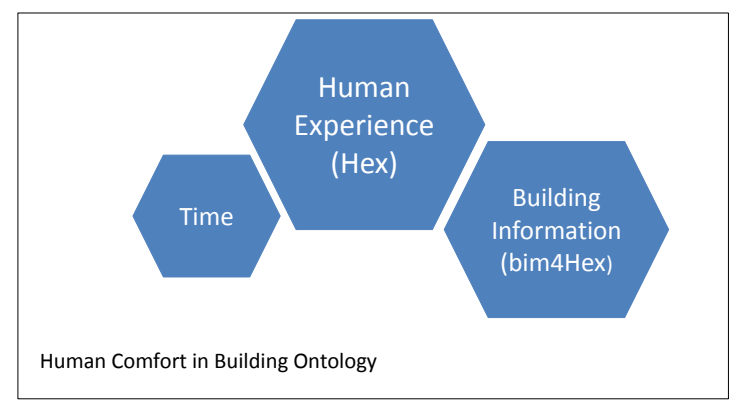

Figure 1. Modules of HBC ontology

Competency questions are the set of the questions that the ontology should be able to answer (See Table 1). These competency question are evaluated via specific use-cases.

\subsection{Conceptualization}

As proposed in METHONTOLOGY [13], the domain knowledge is conceptualized into a model that satisfies the ontology requirements in the Conceptualization activity. It is worth mentioning that there is no single correct way to model the domain of interest. There are always alternative design choices.

Figure 2 shows the overall conceptualization of the HBC ontology. This graphical representation has been created using the Graffoo method [15].

\subsubsection{Hex Module}

The concept classification of IEQ factor is shown in Figure 2. Thermal comfort, visual comfort, acoustic comfort and IAQ are the factors considered for IEQ factors, which makes them subclasses of IEQFactor. Also, they 
Table 1. Basic (1-9) and complex (10-11) competency questions.

\section{Competency Questions}

1. What are the spaces where people experienced positive thermal/visual/acoustic comfort and air quality?

2. What are the spaces where people experienced negative thermal/visual/acoustic comfort and air quality?

3. What are the spaces where people experienced neutral thermal/visual/acoustic comfort and air quality?

4. What are the spaces where good/bad/neutral experiences?

5. What are the spaces where people experienced as a quiet space?

6. What are the spaces where people experienced dim light?

7. When do people usually have good experience of space?

8. When do people usually experience positive visual comfort?

9. When do people experience quietness?

10. What are the equipment settings when people feel cold and report negative thermal comfort?

11 . What could be the adjustment of shading device settings when people report negative visual comfort in a certain space?

are primitive classes. Furthermore, IEQFactor can be decomposed into PostitiveIEQFacotr, NegativeIEQFactor and NeutralIEQFactor based on the value of Quality that it connects to via object property hasQuality. Hence, these three subclasses are defined classes.

Figure 3 shows the concept classification tree of sensation. The modeling corresponds to the design of questions in the human experience survey.

The core concept in the Hex module is Experience (see Figure 2). It connects to IEQFactor by object property isAbout. Experience is also a bridge linking the Time module (via hasTime), and the bim4Hex module (via withObjectSetting and hasLocation) with the Hex module.

\subsection{2 bim4Hex Module}

The bim4Hex ontology contains all concepts, properties and axioms related to building information in HBC. According to ontology requirements, window, air terminal, shading device and space heater are the types of building objects considered in the domain, but can be extended when required.

Figure 4 shows the concept classification tree of BuildingSpace. Spaces are defined based on whether they contain certain types of building objects or not, and they are all subclasses of Buildingspace. For example, the concept definitions of SpaceWithHeater and SpaceWithoutHeater are presented in listing 1 and they are written in Manchester OWL syntax [16]. The rest of the subclasses are defined in the same fashion.

Listing 1. The concept definitions of SpaceWithHeater and SpaceWithoutHeater.

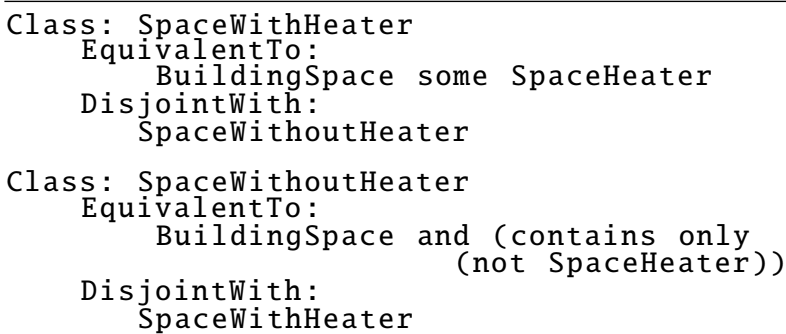

Rules are used to infer rooms that are similar to each other in terms of building objects (see Listing 2). There are nine rules in total. Six rules are omitted here for simplicity, since they are defined in the same fashion as the first two rules. For each type of building objects, there are two rules defined in order to formally specify similar spaces in terms of that specific type. For instance, if two instances are members of class : SpaceHeater, then they are in the relation of : isSimilarSpaceHeaterTo. Similarly, if two instances are members of class : SpaceWithoutHeater, then they are also in the relation of : isSimilarSpaceHeaterTo.

The last rule is defined for inferring spaces that are similar to each other taking all types of building objects into account.

Listing 2. Rules defined for inferring similar rooms in terms of building objects

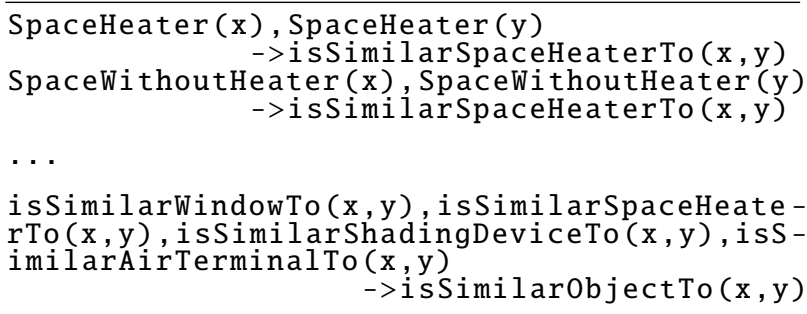

isSimilarWindowTo $(\mathrm{x}, \mathrm{y})$, is SimilarSpaceHeate rTo $(x, y)$, isSimilarShadingDeviceTo $(x, y)$, is imilarAirTerminalTo $(x, y)$ $->$ isSimilarObjectTo $(x, y)$

\subsection{Integration}

\subsubsection{Reusing Existing Ontologies in the Field}

One of the principles for designing an ontology is to reuse ontologies whenever it is possible-this idea has served as our guide here as well.

According to the requirements of the ontology and the human experience survey, we identified three areas where 


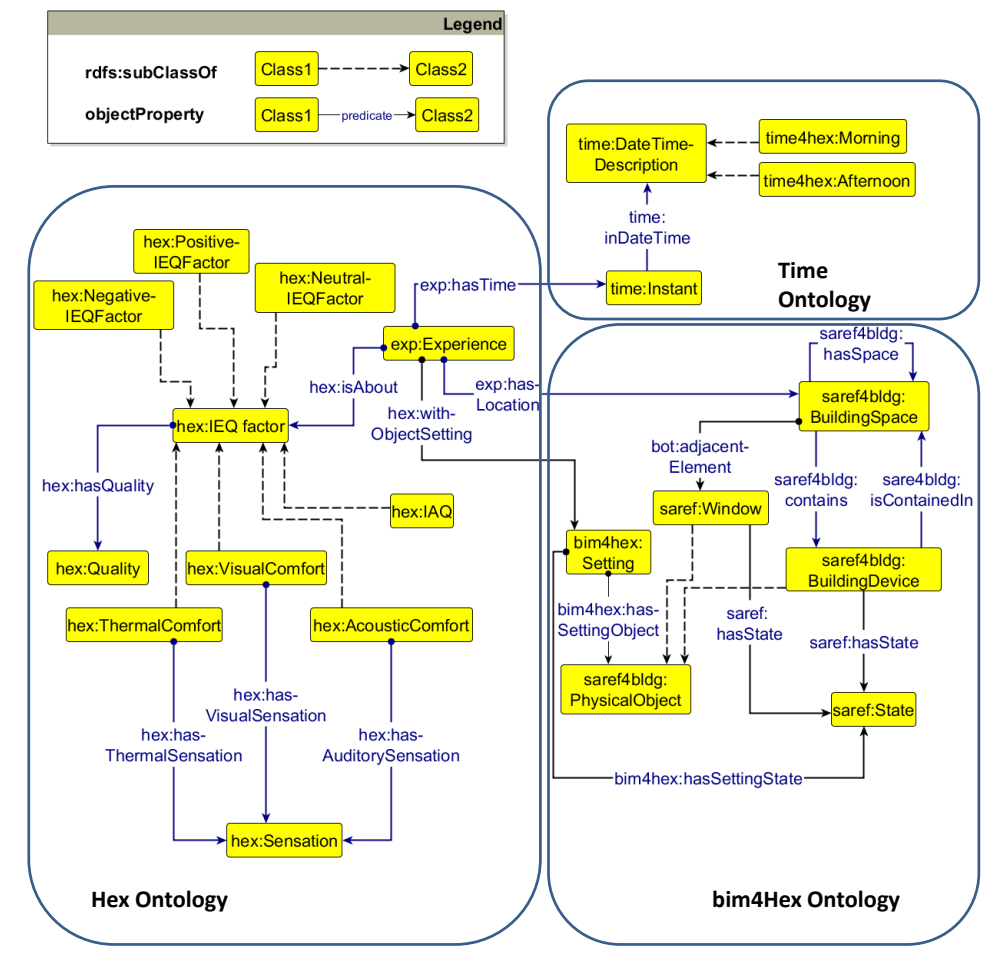

Figure 2. The overview of HBC ontology and its submodules.

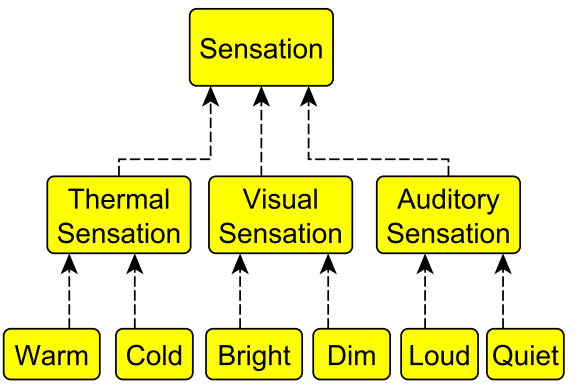

Figure 3. Concept classification tree of Sensation.

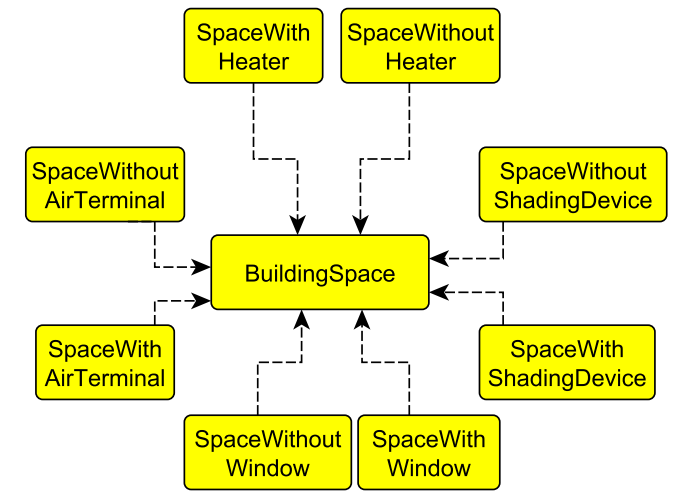

Figure 4. Concept classification tree of BuildingSpace. existing ontologies may be reused. These areas are experiences, building information and time. The Linked Open Vocabularies repository (lov.okfn.org) is used to search desired vocabularies. A brief summery of selected ontologies of the current research domain is presented below:

- Experience Vocabulary [6] is used to describe experiences;

- The SAREF (Smart Appliances REFerence) [17] ontology is used for specifying the devices in a space where experiences are provided;

- The $S 4 B L D G$ ontology [18] is an extension of the SAREF ontology. It is created based on the Industry Foundation Classes (IFC) standard for building information. In our approach, it is used for specifying the building information about a space that experiences are about;

- The BOT (Building Topology Ontology) is an ontology "covering only the core concepts of a building" [19]. It provides extra vocabularies for describing the building elements which are not included in the $S 4 B L D G$ ontology, for example the vocabulary describing the relation between windows and building spaces; 
- OWL-Time [20] specifies temporal properties and it is a W3C candidate recommendation. Currently, although this is a draft version and may be updated in future, the main concepts and properties are likely to remain unchanged;

For this article we surveyed also other ontologies about building information, such as ThinkHome [21], DogOnt ontology [22], ifcOWL ontology [23] and Brick schema [24]. However, in terms of the coverage of concepts based on ontology requirements, $S 4 B L D G$ ontology contains major elements that we needed to for the work reported in this paper.

\subsubsection{Reused Ontology Fragments}

We reuse fragments from the mentioned ontologies as follows. From the Experience vocabulary, we use the concept : Experience and the object properties : hasTime and : hasLocation. From the $S 4 B L D G$ ontology, we use the concepts :BuildingSpace, :PhysicalObject, :BuildingDevice, :SpaceHeater, :ShadingDevice and the object properties : contains and :hasSpace. Furthermore, after the evaluation of the reusable ontologies, some of the terms used in the human experience survey are replaced by corresponding terms in existing ontologies. : AirTerminal is defined in the if $O W L$ ontology [23].

We reuse from the SAREF ontology the concepts :Window, :State, :OnOffState, :OpenCloseState, :ShadingDevice and the object properties :hasState and :hasSpace. We make also use of :adjacentElement from the BOT ontology and :DateTimeDescription, :Instant and the object properties :inDateTime from the OWL-Time ontology. Moreover, the data properties :year,:month, : day, : hour and :minute are also reused.

\subsection{Implementation}

The ontology was developed using the Protégé editor 5.2. Protégé is extensible and provides a plug-and-play environment. Its editor supports editing SWRL rules and importing ontologies. HermiT [25] supports reasoning with SWRL rules and it is available as a Protégé plugin. The implementation of the HBC ontology followed a practical guide presented in [26]. Furthermore, we have taken into account the common errors for modeling with OWL DL pointed out by [27].

The collected data of the human experience survey and building information data is converted into OWL from CSV using OWL API. The ontology is published online ${ }^{1}$.

1https://github.com/TechnicalBuildingSystems/ Ontologies/tree/master/hbc

\subsection{Evaluation}

Our leading principle has been that the evaluation is not only done at the very end of the process of the development. Rather, it is performed in each phase and between activities of the life cycle. If any mismatch is found, the activities of conceptualization, integration, implementation and evaluation will be performed once more.

In this section we evaluate the correctness of :isSimilarObjectTo object property defined using SWRL rules with sample data presented in Table 2.

Table 2. Sample data to evaluate similar rooms (R) in terms of their equipment.

\begin{tabular}{lllll}
\hline $\begin{array}{l}\text { Room } \\
\text { Name }\end{array}$ & $\begin{array}{l}\text { Air } \\
\text { Ter- } \\
\text { minal }\end{array}$ & $\begin{array}{l}\text { Space } \\
\text { Heater }\end{array}$ & $\begin{array}{l}\text { Shading Window } \\
\text { Device }\end{array}$ \\
\hline R16.2.28 & X & & & \\
R16.3.29 & X & & & \\
R16.2.05 & X & X & & \\
R16.3.05 & X & X & & \\
R16.2.11 & & X & X & X \\
R16.3.11 & & X & X & X \\
R16.2.10 & X & X & X & X \\
R16.3.32 & X & X & X & X \\
\hline
\end{tabular}

Figure 5 shows the obtained results after executing the SWRL rules. Among all inference results the reasoner correctly inferred that : Room16.2.05 is similar to itself and : Room16.3.05 in terms of building objects.

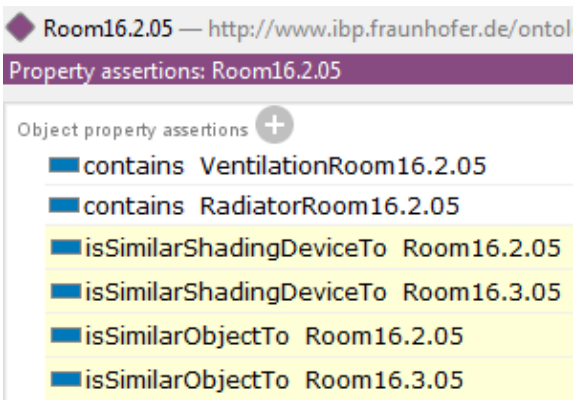

Figure 5. The results after executing the SWRL rules in Protégé.

\section{Use-Case}

We evaluate the ontology by means of answering all of the competency questions (see Table 1). Essentially, those questions can be categorized into two use cases: Space recommendation (1-6) and Setting recommendation (1011). Question 7-9 are about the time feature of experience which further specifies both of the use cases. Hence, all 
the competency questions are covered by these two use cases. In this section, we describe the use case scenarios, the related queries and results.

\subsection{Space Recommendation}

\subsubsection{Scenario}

Often in our daily life we would like to find a certain kind of a space to meet the situation at hand. For example, for studying a textbook,say, in the morning, one might prefer a quiet space. In order to address this need the straightforward question one might ask is, "which spaces are quiet in the morning?"

\subsubsection{Query and Result}

In order to retrieve answers to the question of finding a quiet space, we envision a SPARQL query depicted in Listing 3. ?QuietPercent is the percentage of experiences about acoustic comfort assessed as quiet.

Listing 3. SPARQL query for recommending quiet spaces in the morning.

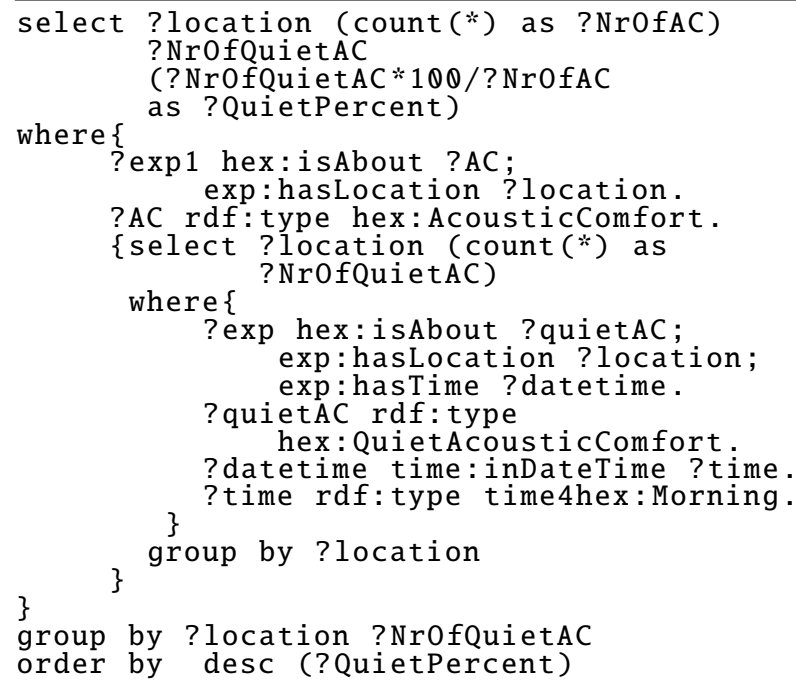

Figure 6 shows the result of the above query with the experimental data obtained through a survey conducted in an office building. According to the chart, Room16.2.20 is the space that receives the highest percentage of experience feedbacks about quiet acoustic comfort in the morning. Consequently, this room seems to be recommendable in a first attempt.

The competency questions $1-6$ can be verified by similar SPARQL queries. It is only necessary to replace the type of ?quietAC, ?AC and ?time by changing the part after rdf: type.

\subsection{Setting Recommendation}

\subsubsection{Scenario}

Equipment settings can be recommended to occupants depending on their comfort requests, if the system that exploits the Hex ontology is integrated with a building control system. For example, if an occupant experiences that the light is too bright in a certain space and requests for a dim light, then the system can recommend settings for the controllable devices in order to provide better visual comfort. The query corresponding to this example is "what kind of shading device settings can be applied for providing positively dim light in a space?".

\subsubsection{Query and Result}

The query shown in Listing 4 is about obtaining the objects and their related states when the occupants report their positive visual comfort with dim visual sensation. The rooms which are similar to each other in terms of objects can be retrieved by property : isSimilarObjectToinferred using the mentioned SWRL rules. Figure 7 shows the result of this query.

Listing 4. SPARQL query for recommending settings of dim visual comfort in the morning.

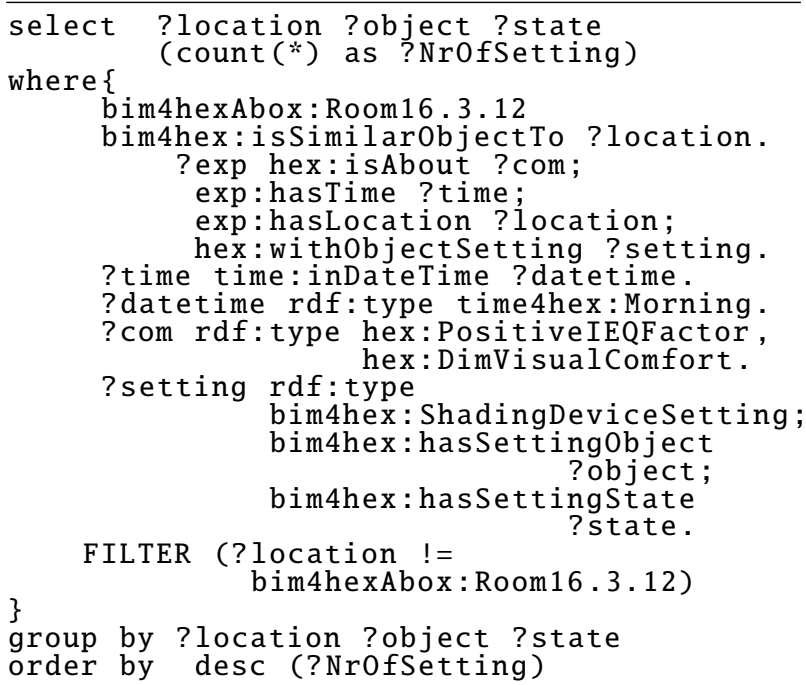

The presented query corresponds to the competency question 11. Similarly, the query can be designed for question 10 by changing the types of ?com and ?setting. The query and result for competency question 10 are omitted here.

\section{Discussion}

We evaluated the ontology in two use cases by means of SPARQL queries. As we reported the results are promising. 


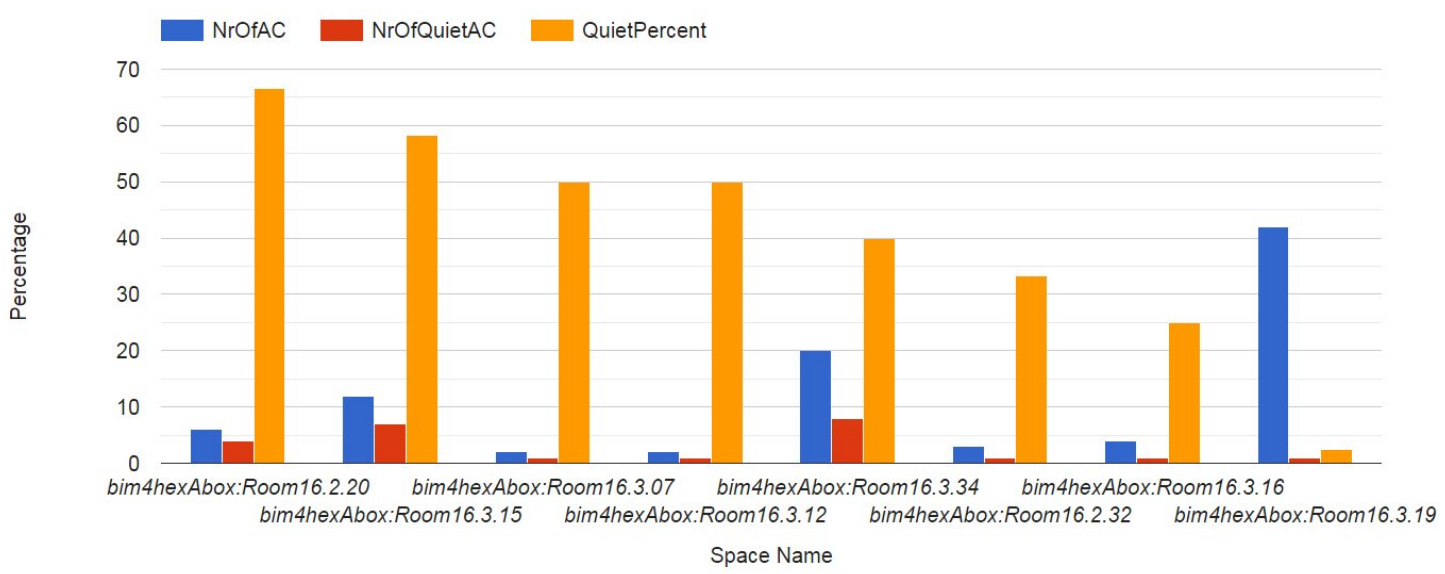

Figure 6. Quiet spaces in the morning.

\begin{tabular}{cclc}
\hline Location & Object & \multicolumn{1}{c}{ State } & NrOfSetting \\
\hline R16.3.15 & WindShutR16.3.15 & PartlyDeployedStatelnst & 3 \\
R16.3.19 & WindShutR16.3.19 & FullyDeployedStatelnst & 2 \\
R16.3.19 & WindShutR16.3.19 & PartlyDeployedStatelnst & 2 \\
R16.3.15 & WindShutR16.3.15 & FullyDeployedStatelnst & 1 \\
R16.3.16 & WindShutR16.3.16 & FullyDeployedStatelnst & 1 \\
R16.3.22 & WindShutR16.3.22 & NotDeployedStatelnst & 1 \\
\hline
\end{tabular}

Figure 7. Ordered recommended settings for positively dim light.

However, we have identified some limitations. First of all, ontology serves merely as an aid for the initial step for analyzing data. Further on, data analysis techniques are required when one desires more profound and rigid results. This is in particular the case when evaluating quantitative or time-encoded data. For instance, an experience about a room from one day ago should in an extended analysis treaded differently than an experience from last year (see the first use-case).

Furthermore, although human experiences can reveal some facts of the reality, it is not sufficient to utilize human experiences alone in an objective way. The great strength comes from combining human experiences within the context of more objective information, such as sensor observations, weather information and so on.

\section{Conclusion}

Understanding human experiences of Indoor Environmental Quality (IEQ) has potential benefits including reducing energy demand and enhancing occupants' comfort. We provide an ontology for capturing human experiences about the several dimensions of IEQ in spaces by means of which we confirm the following hypotheses:

- Human experiences about IEQ factors can provide insights about their spaces;

- Human experiences about IEQ factors can inform building settings.

The ontology consists of three modules: Hex, bim4Hex and Time. It formally defines the domains of IEQ, experiences and their relationship to Building Information Modelling (BIM) and time.

In order to evaluate the HBC ontology, we collected data through designed human experience survey and converted them into the Resource Description Format (RDF) compliant with HBC ontology. With the help of reasoning, all desired implicit data were obtained. SPARQL queries were designed for answering questions based on two identified use cases corresponding the hypotheses. The queries performed over the gathered data are able to provide expected results. This supports a conclusion that $\mathrm{HBC}$ has the ability to leverage the Semantic Technologies to provide insights of spaces and inform building settings.

With large sources of human experiences, such as hotel reviews written in text, the system can provide the advanced searching options based on IEQ, e.g. quiet rooms or rooms with good visual comfort. In addition, the ability to predict human IEQ could assist towards more informative control decisions in specific Building Management Systems.

\section{Acknowledgements}

The authors gratefully acknowledge the use of services and facilities at the Energie Campus Nürnberg, the financial support by the "Aufbruch Bayern (Bavaria on the move)" initiative of the state of Bavaria, and the financial support by the Federal Ministry for Economic Affairs and Energy of Germany (BMWi) in the project ValMoNuI (project number 03ET1289C). Responsibility for the content of this publication lies with the authors. 


\section{References}

[1] Bentayeb M. and Simoni M. et al. Indoor air pollution and respiratory health in the elderly. Environmental Science and Health, 48:1783-1789, 2013.

[2] Agha-Hossein M. M. and El-Jouzi S. et al. Postoccupancy studies of an office environment: Energy performance and occupants' satisfaction. Building and Environment, 69:121-130, 2013.

[3] Fanger P. O. Thermal comfort. Danish Technical Press, Copenhagen, 1970.

[4] Nicol J. F. and Roaf S. Rethinking thermal comfort. Building Research \& Information, 72:1-5, 2017.

[5] Park J. Are Humans Good Sensors? Using Occupants as Sensors for Indoor Environmental Quality Assessment and for Developing Thresholds that Matter. PhD thesis, Carnegie Mellon University, Pittsburgh, USA, 2015.

[6] Kauppinen T., Litvinova E., and Kallenbach J. Capturing and Linking Human Sensor Observations with Yousense. In Proc. of ISWC, volume 1272, pages 373-376, Riva del Garda, Italy, 2014.

[7] Building Robotics' Comfy. Comfy. on-line: https://www.comfyapp.com/, Accessed:01/02/2018.

[8] Pritoni M. and Salmon K. et al. Occupant thermal feedback for improved efficiency in university buildings. Energy and Buildings, 144:241-250, 2017.

[9] CrowdComfort Inc. Crowdcomfort. on-line: http://crowdcomfort.com/, Accessed:01/02/2018.

[10] Zagreus L. and Huizenga C. et al. Listening to the occupants: a Web-based indoor environmental quality survey. Indoor Air, 14:65-74, 2004.

[11] Huber C., Eitzinger S., and Koch D. Measuring user satisfaction. In Proc. EuroFM, pages 121-129, Milan, Italy, 2016.

[12] Corry E. and Donnell J. et al. Using semantic web technologies to access soft AEC data. Advanced Engineering Informatics, 28:370-380, 2014.

[13] Lopez M. F. and Perez A. G. et al. METHONTOLOGY: from ontological art towards ontological engineering. In Proc. of AAAI, pages 33-40, Stanford, USA, 1997.

[14] Simperl E., Mochol M., and Bürger T. Achieving maturity: The state of practice in ontology engineering in 2009. International Journal of Computer Science and Applications, 7:45-65, 2010.
[15] Falco R., Gangemi A., and Peroni S. et al. Modelling OWL ontologies with Graffoo. In Proc. of ESWC, pages 320-325, Anissaras, Greece, 2014.

[16] Horridge M. and Patel P. OWL 2 Web Ontology Language Manchester Syntax (Second Edition). On-line: http://www.w3.org/TR/2012/NOTEowl2-manchester-syntax-20121211/, Accessed: 02/02/2018.

[17] Daniele L., den Hartog F., and Roes J. Created in close interaction with the industry: The Smart Appliances REFerence (SAREF) Ontology. In Proc. of FOMI, pages 100-112, Berlin, Germany, 2015.

[18] Poveda V. M. and Garcia C. R. SAREF extension for building devices. On-line: http://ontoology.linkeddata.es/publish/saref4bldg/index-en.html, Accessed: 2017-08-027.

[19] Rasmussen M. H., Pauwels P., Karlshøj J., and Hviid C. Proposing a central AEC ontology that allows for domain specific extensions. In Proc. of LC3, pages 237-244, Heraklion, Greece, 2017.

[20] Cox S. and Little C. Time ontology in owl. Online: https://www.w3.org/TR/owl-time/, Accessed: 01/08/2017.

[21] Reinisch C. and Kofler M. J. et al. ThinkHome energy efficiency in future smart homes. EURASIP Journal on Embedded Systems, pages 1-18, 2011.

[22] Bonino D. and Corno F. DogOnt - ontology modeling for intelligent domotic environments. In Proc. of ISWC, pages 790-803, Karlsruhe, Germany, 2008.

[23] Pauwels P., Krijnen T., Terkaj W., and Beetz J. Enhancing the ifcOWL ontology with an alternative representation for geometric data. Automation in Construction, 80:77-94, 2017.

[24] Balaji B. et al. Brick: Metadata schema for portable smart building applications. Applied Energy, 2018.

[25] Shearer R., Motik B., and Horrocks I. HermiT: A highly-efficient OWL reasoner. In OWLED, volume 432, page 91, 2008.

[26] Horridge M. and Knublauch H. et al. A Practical Guide To Building OWL Ontologies Using The Protege-OWL Plugin and CO-ODE Tools Edition 1.3, 2011.

[27] Rector A., Drummond N., and Horridge M. et al. OWL Pizzas: Practical experience of teaching OWLDL: Common errors \& common patterns. In Proc. of EKAW, pages 63-81, Whittlebury Hall, UK, 2004. 